\title{
Simposio
}

\section{Parasitología Veterinaria}

\section{Garrapatas: estado actual y perspectivas}

\author{
Jesús Alfredo Cortés-Vecino
}

Grupo de Parasitología Veterinaria, Laboratorio de Parasitología Veterinaria, Universidad Nacional de Colombia, Bogotá D.C., Colombia

Diferentes especies de artrópodos tienen importancia veterinaria por causar enfermedades, transmitir organismos patógenos a los animales y generar pérdidas económicas significativas. Entre éstos, las garrapatas y las enfermedades transmitidas por las mismas, constituyen una de las principales limitantes de la industria bovina en el mundo. El impacto económico negativo se debe, esencialmente, a los detrimentos directos sobre la producción; a la extracción de volúmenes considerables de sangre; a daños en las pieles de los animales por heridas locales, que pueden ser secundariamente colonizadas por bacterias o por larvas de moscas; a efectos tóxicos y alteraciones en la producción de leche y carne; a la disminución en la fertilidad; a estados de anorexia que conllevan a un mayor tiempo de crecimiento y ceba de los animales, y a dificultades en la adaptación al ambiente tropical de razas mejoradas o importadas para incrementar la calidad genética y la productividad de las ganaderías.

Las garrapatas son ectoparásitos hematófagos obligados, prácticamente de todos los vertebrados terrestres (principalmente, mamíferos), aves, reptiles y algunos anfibios, y aunque han sido considerados parásitos cosmopolitas, numerosas especies están restringidas a regiones (hábitats) específicas $(1,2)$. Para agrupar taxonómicamente a las garrapatas, se han reconocido tres familias (Ixodidae, Argasidae y Nuttalliellidae), dentro de la superfamilia Ixodoidea, a su vez incluida dentro del suborden Ixodida. No obstante, la clasificación taxonómica de estos artrópodos y la ubicación de algunas especies dentro de un taxón determinado, con frecuencia han generado debates.

Las herramientas moleculares actuales han permitido grandes avances científicos en el entendimiento de las relaciones filogenéticas de las garrapatas. Sin embargo, no existe unanimidad sobre los cambios propuestos en la sistemática de estos artrópodos, por lo que algunas reubicaciones taxonómicas no son completamente aceptadas. En este sentido, los especialistas hacen uso de caracteres que comprenden forma (morfología) y función (aspectos tales como biología, ecología y comportamiento). Es más, se espera que en los próximos años se precisen nuevas relaciones filogenéticas, al margen del consenso de expertos e investigadores (2-4).

Tomando en cuenta lo anterior, se reconocen alrededor de 907 nombres científicos válidos de géneros y especies de garrapatas distribuidas en todo el mundo, especialmente concentradas en las regiones tropicales y subtropicales (4). Un diagrama con la distribución taxonómica reconocida de las familias y subfamilias de las garrapatas se presenta en la figura 1.

Recientemente, entre las nuevas relaciones filogenéticas establecidas, debe destacarse la del género Boophilus (Curtice, 1891), el cual se ha convertido en subgénero del género Rhipicephalus (Koch, 1844), por ser este último parafilético con respecto al primero; por lo tanto, las especies de garrapatas que pertenecían al género Boophilus han sido reclasificadas recientemente como un subgénero del género Rhipicephalus a partir de evidencias moleculares, citogenéticas, morfológicas, biogeográficas y de interacción con los huéspedes $(5,6)$.

En Colombia, las primeras citas sobre garrapatas aparecen en descripciones de las colecciones entomológicas de Osorno-Mesa (1939). En su publicación "Las Garrapatas de la República de Colombia" se incluye una descripción de las especies identificadas, sus huéspedes y lugares de presentación, al igual que una clave taxonómica para la identificación del género y las especies de garrapatas recolectadas (7). 


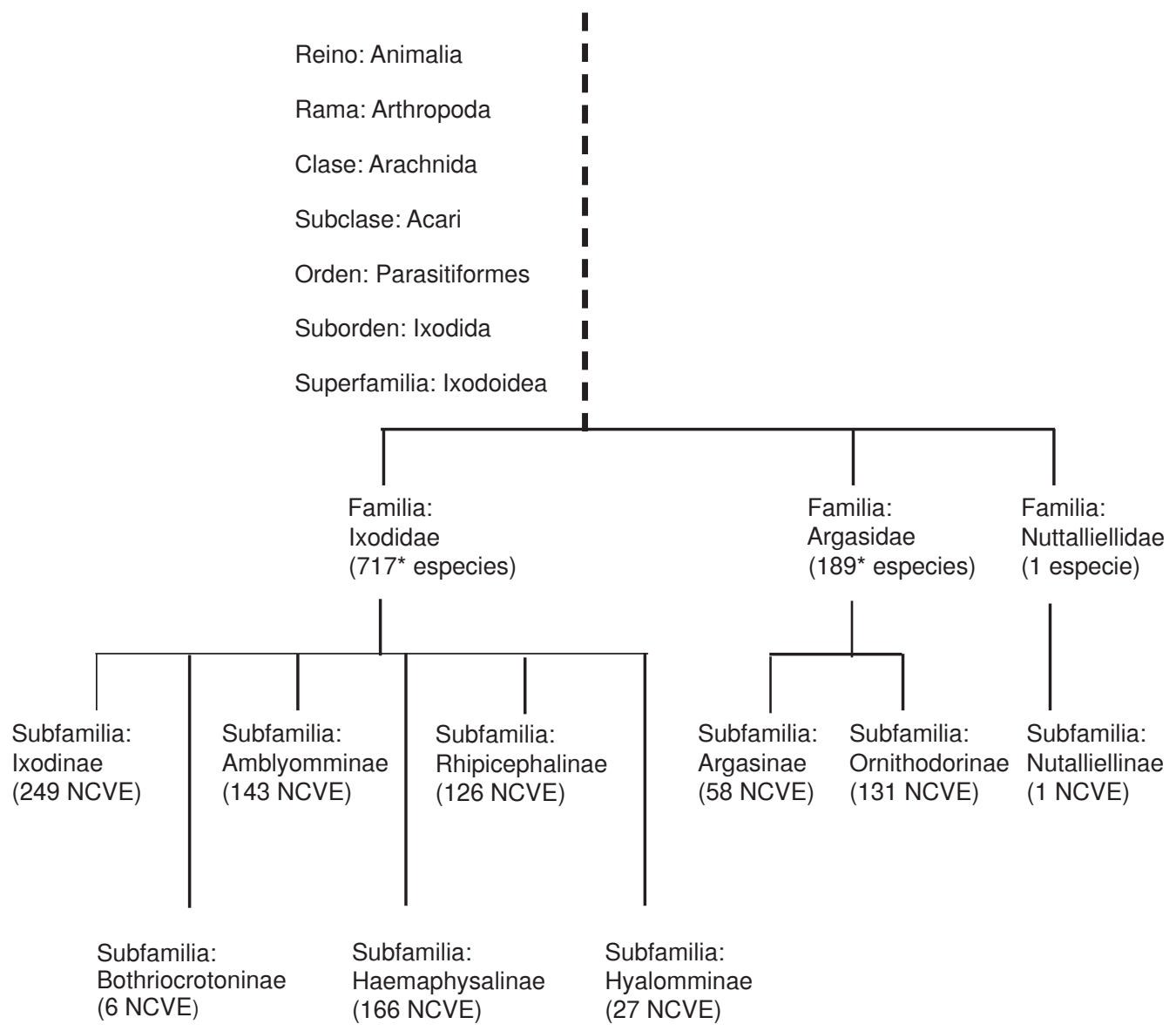

Fuente: Barker y Murrel, 2008; Dantas-Torres, 2008.

NCVE: nombres científicos válidos de especies

Figura 1. Distribución taxonómica de familias y subfamilias de garrapatas con el número de especies válidas que contienen.

El ciclo de vida de las garrapatas incluye cuatro estadios: huevo, larva, ninfa y adulto. Las garrapatas ixódidas solamente presentan un estadio de ninfa, en tanto que las garrapatas argásidas tienen dos o más estadios de ninfa (8). El ciclo de vida de las garrapatas ixódidas puede ser de uno hasta tres huéspedes (9) (figura 2).

Las garrapatas poseen importancia sanitaria y económica debido al daño directo que causan al alimentarse para extraer sangre de su huésped, a la transmisión de microorganismos patógenos como bacterias, rickettsias, protozoarios, virus y hongos (y nematodos experimentalmente), y a la inoculación de sustancias tóxicas a sus huésped $(1,2,12)$. Están consideradas como el segundo grupo de vectores (después de los mosquitos) de importancia en la transmisión de enfermedades infecciosas a humanos y animales en el ámbito mundial $(10,11)$.

La capacidad vectorial de las garrapatas de la familia Ixodidae se ve favorecida por varios aspectos. En primer lugar, se alimentan por períodos relativamente largos durante los cuales permanecen firmemente adheridas al huésped y no se sueltan fácilmente. En segunda instancia, la picadura, usualmente indolora, pasa desapercibida hasta que la garrapata se ingurgita completamente de sangre (11). Como tercer punto, el ambiente en el intestino medio de las garrapatas es menos hostil que el de insectos hematófagos, ya que poseen proteasas intracelulares; en consecuencia, los patógenos ingeridos no están expuestos directamente a la acción proteolítica de estas enzimas $(10,11)$. Esta característica les da ventajas 

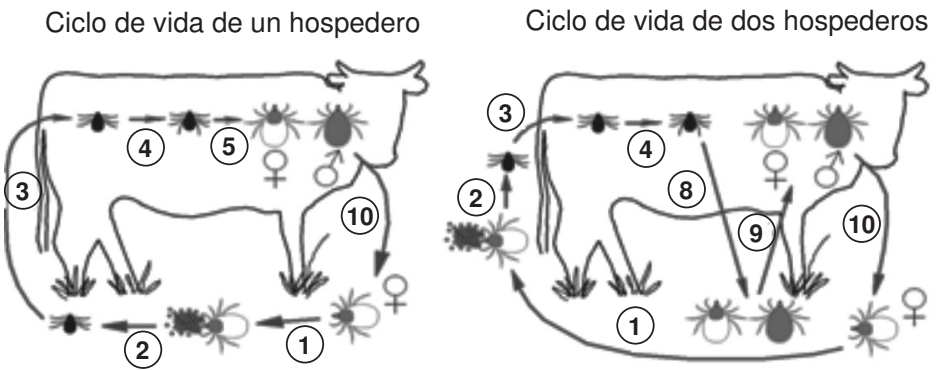

Ciclo de vida de tres hospederos

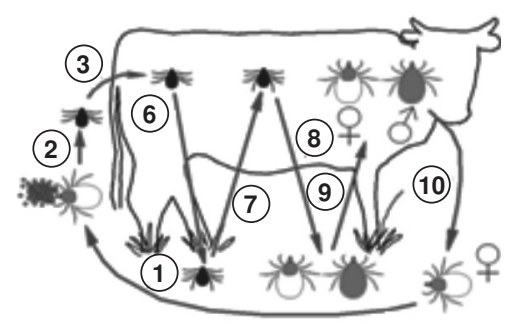

Figura 2. Ciclos de vida para ixódidos de uno, dos y tres huéspedes

Elaborada por: Forero-Becerra, 2011.

1: oviposición; 2: eclosión de los huevos; 3: larvas buscan y se adhieren a un huésped; 4: muda de larva a ninfa sobre el huésped; 5: muda de ninfa a adulto sobre el huésped; 6: muda de larva a ninfa fuera del huésped; 7: ninfas buscan y se adhieren a un huésped; 8: muda de ninfa a adulto fuera del huésped; 9: adultos buscan y se adhieren a un huésped; 10: hembra apareada e ingurgitada se desprende.

como transmisores de agentes patógenos y ubica a las garrapatas en los artrópodos que mayor cantidad de organismos transmiten $(10,11)$.

Entre las enfermedades que transmiten las garrapatas como vectores, se encuentran las bacteriosis (fiebre $Q$, bartonelosis, tularemia), enfermedades por rickettsias (anaplasmosis, ehrlichiosis y fiebres manchadas, entre otras), las borreliosis (enfermedad de Lyme y fiebre recurrente, entre otras) (11). Además, transmiten varias enfermedades causadas por protozoarios (babesiosis y theileriosis, entre otras) y virus (fiebre del Colorado, encefalitis transmitida por garrapatas, encefalomielitis infecciosa de las ovejas o Louping III y peste porcina africana, entre otros). Se reporta transmisión de hongos, como ocurre con Dermatophilus congolensis por la garrapata Amblyomma variegatum (8). $\mathrm{Y}$ existe evidencia experimental de la transmisión de nematodos, tipo filarias, como Dipetalonema dracunculoides por parte de Rhipicephalus sanguineus (12).

Además, la presencia de una neurotoxina en la saliva de las garrapatas hembra en alimentación (55 especies de la familia Ixodidae y 14 especies de la familia Argasidae) puede causar una enfermedad conocida como "parálisis por garrapatas". La toxina interrumpe la transmisión sináptica de los nervios motores de la columna vertebral y bloquea las uniones neuromusculares al impedir la liberación de acetilcolina y así causa daño a los sitios receptor. Entre los signos se incluyen: disfunción nerviosa periférica, dificultad respiratoria, efectos cardiovasculares, vómito y cambios en la temperatura corporal, y es considerada una importante causa de muerte en bovinos, ovinos y caprinos en varias regiones del mundo $(13,14)$.

Las pérdidas económicas por garrapatas fueron estimadas en US\$ 7 billones en todo el mundo, y de esos, un billón de dólares corresponde a Latinoamérica (15). Las pérdidas y los perjuicios que ocasionan en los diferentes sistemas de producción pecuaria y en salud pública y animal, hacen necesaria la búsqueda permanente de alternativas y medidas eficientes de tratamiento y control.

Los programas de control integrado de garrapatas están encaminados a lograr un uso óptimo de los productos (tradicionales y de nueva generación) que aún son efectivos, mediante la integración de herramientas y estrategias, químicas y no químicas, para el control de garrapatas. La resistencia de diferentes especies de garrapatas a los productos garrapaticidas existe incondicionalmente en la mayor parte de las regiones del mundo, donde se ha realizado su control químico y es inevitablemente una consecuencia del uso de parasiticidas (2).

Entre las tendencias actuales y futuras de la investigación en garrapatas se encuentran los análisis taxonómicos y filogenéticos; los estudios de biología molecular y genética de poblaciones; los estudios de distribución y favorabilidad del hábitat; las investigaciones de interacciones con patógenos y su relación con la epidemiología de enfermedades de importancia en salud pública y salud animal; los estudios de caracterización y extensión de la resistencia a garrapaticidas; los programas y alternativas de manejo integrado, así como el desarrollo de vacunas contra las garrapatas y el mejoramiento genético de los huéspedes.

\section{Referencias}

1. Guglielmone A, Bechara G, Szabó M, Barros D, Faccini J, Labruna MB, et al. Garrapatas de importancia médica y veterinaria: América Latina y EI Caribe. The Netherlands; 2004.

2. Pereira MC, Labruna MB, Szabó MPJ, Klafke GM. Rhipicephalus (Boophilus) microplus: biologia, 
controle e resistência. São Paulo: MedVet Livros; 2008.

3. Dantas-Torres F. The brown dog tick, Rhipicephalus sanguineus (Latreille, 1806) (Acari: Ixodidae): from taxonomy to control. Vet Parasitol. 2008;152:17385.

4. Barker S, Murrell A. Systematics and evolution of ticks with a list of valid genus and species names. In: Bowman A, Nutall P, editors. Ticks: biology, disease and control. Cambridge: Cambridge University Press; 2008. p. 1-39.

5. Murrell A, Campbell NJ, Barker SC. Phylogenetic analyses of the Rhipicephaline ticks indicate that the genus Rhipicephalus is paraphyletic. Mol Phylogenet Evol. 2000;161-7.

6. Murrell A, Barker SC. Synonymy of Boophilus Curtice, 1891 with Rhipicephalus Koch, 1844 (Acari: Ixodidae). Syst Parasitol. 2003;56:169-72.

7. Osorno-Mesa E. Las Garrapatas de la República de Colombia: Academia Nacional de Medicina,1939. Comunicación en la sesión del 14 de Noviembre de 1939.

8. Nicholson W, Sonenshine D, Lane R, Uilenberg G. Ticks (Ixodida). In: Mullen G, Durden L, editors. Medical and Veterinary Entomology. San Diego: Academic Press; 2009. p. 493-542.
9. Ensinas-Grandes A, Oleaga-Pérez A, Pérez-Sánchez R. Garrapatas Duras. In: Cordero del Campillo MR-V FA, editor. Parasitología veterinaria. Madrid: McGraw Hill-Interamericana de España, SAU; 1999. p. 420-9.

10. Anderson JF. The natural history of ticks. Medical Clin North Am. 2002;86:205-18.

11. Anderson JF, Magnarelli LA. Biology of ticks. Infect Dis Clin North Am. 2008;22:195-215.

12. Olmeda-García AS, Rodríguez-Rodríguez JA, Rojo-Vázquez FA. Experimental transmission of Dipetalonema dracunculoides (Cobbold 1870) by Rhipicephalus sanguineus (Latreille 1806). Vet Parasitol. 1993;47:339-422.

13. Wall R, Shearer D. Ticks (Acari). In: Wall R, Shearer D, editors. Veterinary ectoparasites: biology, pathology, and control. 2 ed. Oxford: Blackwell Science Ltd.; 2001. p. 55-82.

14. Mans BJ, Gothe R, Neitz AWH. Tick toxins: perspectives on paralysis and other forms of toxicoses caused by ticks. In: Bowman AS, Nutall P, editors. Ticks: biology, disease and control. Cambridge: Cambridge University Press; 2008. p. 108-26.

15. Castro-Janer E, Rifran L, Gonzalez P, Piaggio J, Gil A, Schumaker TT. Rhipicephalus (Boophilus) microplus (Acari: Ixodidae) resistance to Fipronil in Uruguay evaluated by in vitro bioassays. Vet Parasitol. 2010;169:172-7.

\title{
Miasis en salud pública y salud pública veterinaria
}

\author{
Elkin Gustavo Forero-Becerra
}

Grupo de Parasitología Veterinaria, Laboratorio de Parasitología Veterinaria, Universidad Nacional de Colombia, Bogotá, D.C., Colombia

\section{Aspectos generales de las moscas responsables de miasis}

El orden Diptera comprende más de 120.000 especies descritas y es uno de los órdenes con mayor número de especies en la clase Insecta (1). En el orden Diptera, el infraorden Muscomorpha ha sido implicado con mayor frecuencia en los casos de miasis humana y animal. Para describir la presencia de larvas en tejidos de animales y humanos, Hope (2) propuso el término miasis (del griego, myia: mosca; -iasis, sufijo para los nombres de enfermedades). Poco más de un siglo después, Zumpt (3) definió la miasis como la infestación de humanos y animales con larvas de dípteros, los cuales, al menos por un período, se alimentan de los tejidos vivos o muertos del huésped, de líquidos corporales o de alimentos ingeridos.

La clasificación taxonómica de los géneros de moscas frecuentemente reportados en la literatura como responsables de miasis en humanos y animales se presenta en la figura 1.
Por otro lado, las miasis han sido clasificadas de varías formas para facilitar la comprensión de su patogenia, tratamiento y medidas de control. Una síntesis de los diferentes tipos de clasificación se presenta en la tabla 1.

\section{Epidemiología de las miasis}

Con frecuencia, las miasis suelen ser causadas por especies oportunistas, donde la infestación depende de varios factores:

1. la presencia de moscas hembras grávidas en busca de un sustrato de oviposición;

2. altos niveles de exposición de un huésped potencial susceptible a las moscas que causan miasis por deficientes condiciones de higiene, áreas expuestas de la piel normalmente cubiertas o inapropiado tratamiento de heridas (con frecuencia asociados a indigencia en humanos);

3. dormir a la intemperie;

4. agresividad incrementada de las moscas que causan miasis; 
5. distribución geográfica; y

6. la época del año (particularmente en verano) $(4,5)$.

De esta manera, algunos casos de miasis humana son accidentales (6) o se encuentran asociados a incapacidad de defensa del paciente (7), enfermedades concomitantes y parasitosis externa. Sin embargo, las moscas responsables de miasis obligatoria suelen tener un rango de huéspedes definido, un sitio específico de localización anatómica y una distribución geográfica limitada.

De acuerdo con Cassie, et al. (4), los principales grupos de riesgo para miasis en humanos están constituidos por indigentes, ancianos o personas débiles. En animales de producción, los casos de miasis están asociados, principalmente, con condiciones y actividades de manejo, tipo de producción o susceptibilidad de raza (8). En animales de compañía, las miasis se consideran accidentales, particularmente como consecuencia de heridas abiertas (9). En animales silvestres, las moscas de miasis actúan como agentes de control biológico oportunistas o son parásitos específicos de especie.

La mayoría de los reportes de miasis en la literatura se originan en países tropicales en desarrollo o se han detectado en países industrializados a partir de personas provenientes de regiones tropicales y subtropicales. No obstante, existen reportes de miasis nativas en los países desarrollados (4) y su frecuencia podría aumentar en los años venideros.

\section{Retos para el médico veterinario en Colombia}

Varias especies de miasis obligatoria, facultativa y accidental se encuentran en Colombia. ForeroBecerra et al. (10) hallaron que la miasis traumática obligatoria por Cochliomyia hominivorax constituye un serio problema en las fincas de ganadería bovina que realizan prácticas de manejo como castración, marcación y descornado. A través

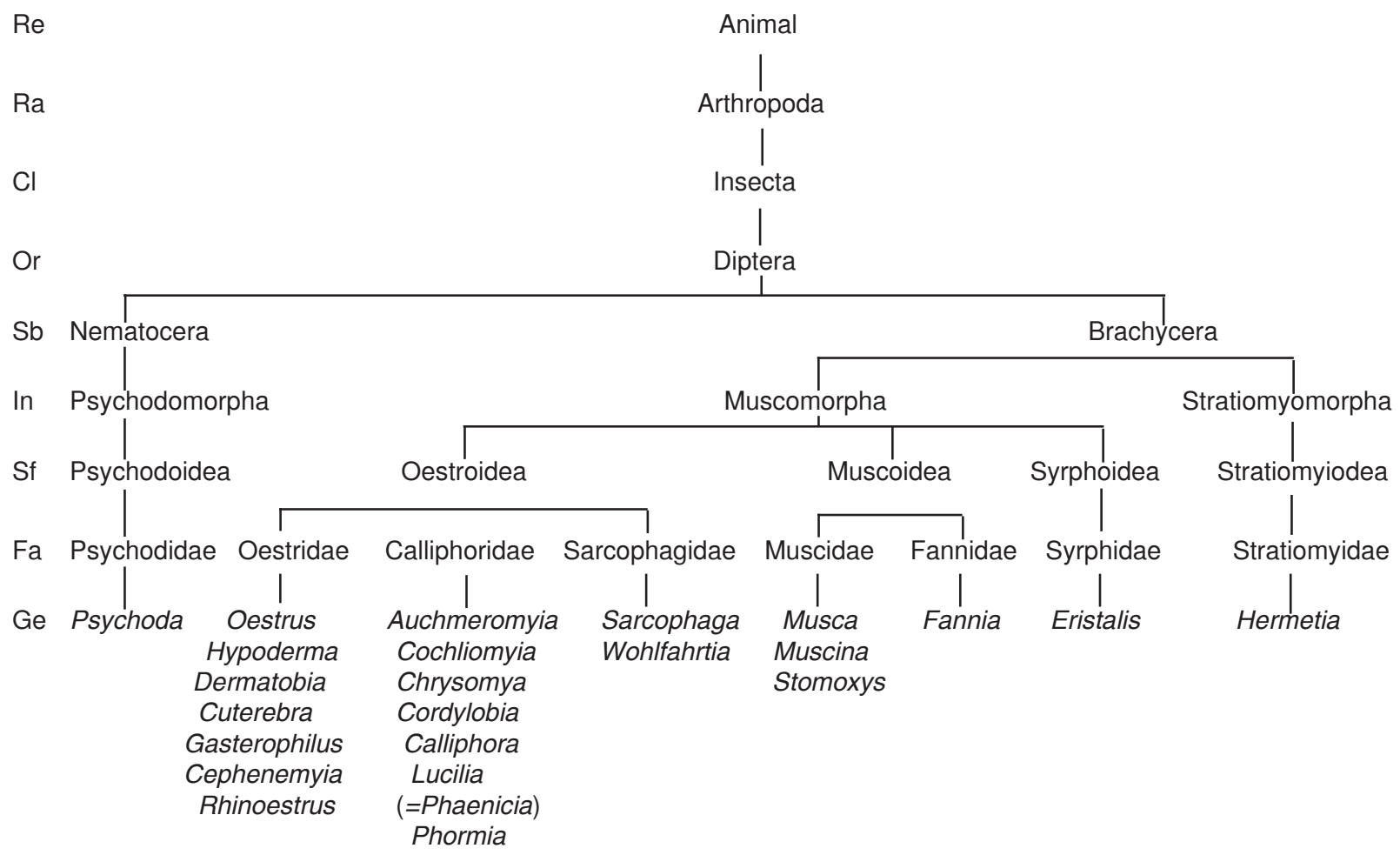

* También se han reportado especies de otros géneros como Megaselia, Dryomyza, Drosophila, Thereva, Piophila, Neottiophilum, Batrachomyia, Hylemya, Hydrotaea, Passeromyia, Mydaea, Neomusca, Bufolucilia, Eucalliphora, Paralucilia, Protophormia, Cynomya, Protocalliphora, Pachychoeromyia, Booponus, Elephantoloemus, Anolisomyia, Cistudinomyia, Notochaeta, Eumachronychia, Metoposarcophaga, Ochotonia, Oestromyia, Oestroderma, Pallisiomyia, Pavlovskiata, Portschinskia, Przhevalskiana, Strobiloestrus, Cephalopina, Gedoelstia, Kirkioestrus, Pharyngobolus, Pharyngomyia, Tracheomyia, Cobboldia, Gyrostigma, Neocuterebra, y Ruttenia.

Re: reino; Ra: rama; Cl: clase; Or: orden; Sb: suborden; In: infraorden; Sf: superfamilia; Fa: familia; Ge: género

Figura 1. Taxonomía de los principales géneros* reportados en miasis de humanos y animales. 
Tabla 1. Tipos de clasificación de las miasis.

\begin{tabular}{|c|c|c|}
\hline Clasificación & Descripción & Comentario \\
\hline \multirow[t]{3}{*}{$\begin{array}{l}\text { Hábitos alimenticios } \\
\text { de las larvas }\end{array}$} & $\begin{array}{l}\text { Biontófagas, se alimentan solo } \\
\text { de tejidos vivos. }\end{array}$ & \multirow{3}{*}{$\begin{array}{l}\text { La discriminación de las larvas por sus } \\
\text { hábitos alimenticios es útil para identificar } \\
\text { las especies de uso potencial en } \\
\text { terapia larvaria. }\end{array}$} \\
\hline & $\begin{array}{l}\text { Necrobióticas, se alimentan de } \\
\text { tejidos muertos. }\end{array}$ & \\
\hline & $\begin{array}{l}\text { Necrobiontófagas, se alimentan de } \\
\text { carroña, pero bajo determinadas } \\
\text { circunstancias, se pueden encontrar } \\
\text { en animales y humanos vivos. }\end{array}$ & \\
\hline \multirow[t]{3}{*}{$\begin{array}{l}\text { Sitio de localización } \\
\text { anatómica }\end{array}$} & $\begin{array}{l}\text { Miasis cutánea } \\
\text { - Foruncular } \\
\text { - Traumática o de las heridas } \\
\text { - Migratoria }\end{array}$ & \multirow{3}{*}{$\begin{array}{l}\text { El sitio de localización anatómica de las } \\
\text { miasis indica la seriedad de la lesión y } \\
\text { facilita la selección del tratamiento más } \\
\text { apropiado } \\
\text { ) }\end{array}$} \\
\hline & $\begin{array}{l}\text { Miasis de orificios naturales (cavidades) } \\
\text { - Auricular (aural o auditiva) } \\
\text { - Ocular } \\
\text { - Nasal } \\
\text { - Oral } \\
\text { - Genital } \\
\text { - Anal }\end{array}$ & \\
\hline & $\begin{array}{l}\text { Miasis de órganos internos } \\
\text { - Intestinal } \\
\text { - Urinaria } \\
\text { - Uterina } \\
\text { - Cerebral }\end{array}$ & \\
\hline Relación con el huésped & $\begin{array}{l}\text { Miasis obligatorias } \\
\text { Miasis facultativas } \\
\text { Miasis accidentales }\end{array}$ & $\begin{array}{l}\text { Esta clasificación define con precisión los } \\
\text { sustratos de desarrollo larvario para } \\
\text { establecer medidas efectivas de prevención } \\
\text { y control. }\end{array}$ \\
\hline Lugar de infestación & $\begin{array}{l}\text { Miasis rural } \\
\text { Miasis urbana } \\
\text { Miasis foránea } \\
\text { Miasis nativa } \\
\text { Miasis hospitalaria }\end{array}$ & $\begin{array}{l}\text { Aunque esta clasificación no es muy } \\
\text { utilizada, permite comprender los factores } \\
\text { de riesgo de las miasis según el grado de } \\
\text { exposición o tipo de actividad. Para el caso } \\
\text { miasis hospitalaria también se debe tener en } \\
\text { cuenta el estado de indefensión. }\end{array}$ \\
\hline $\begin{array}{l}\text { Susceptibilidad o rareza } \\
\text { de infestación del grupo } \\
\text { etario }\end{array}$ & $\begin{array}{l}\text { Miasis neonatal } \\
\text { Miasis geriátrica }\end{array}$ & $\begin{array}{l}\text { Es muy rara, pero útil para llamar la atención } \\
\text { sobre el compromiso del sistema inmunitario } \\
\text { o la capacidad de respuesta del individuo } \\
\text { frente a una infestación por larvas de } \\
\text { dípteros. }\end{array}$ \\
\hline $\begin{array}{l}\text { Daño causado a los tejidos } \\
\text { y compromiso de la salud } \\
\text { del hospedero }\end{array}$ & $\begin{array}{l}\text { Miasis maligna } \\
\text { Miasis autolimitada }\end{array}$ & $\begin{array}{l}\text { Si bien las larvas se desarrollan durante } \\
\text { período de tiempo preciso, en algunos } \\
\text { casos la seriedad de la lesión facilita la } \\
\text { infestación sucesiva de nuevas cargas } \\
\text { larvarias por moscas de la misma especie o } \\
\text { de diferentes especies. Además, el } \\
\text { compromiso de los tejidos afectados, el } \\
\text { grado de infección bacteriana secundaria y } \\
\text { el efecto tóxico de algunos metabolitos de las } \\
\text { larvas pueden ser criterios útiles para esta } \\
\text { clasificación. }\end{array}$ \\
\hline
\end{tabular}


de la técnica de insecto estéril, esta mosca fue erradicada de los Estados Unidos, México y Centroamérica en 40 años, aproximadamente. Sin embargo, recientemente se ha objetado la eficacia del programa de erradicación.

Por otro lado, Dermatobia hominis causa pérdidas económicas significativas a la ganadería vacuna colombiana. Su control con ivermectinas podría tener impactos negativos en la entomofauna coprofílica asociada a las heces bovinas. No se encontraron estudios epidemiológicos recientes de la miasis nasal obligatoria por Oestrus ovis en Colombia, pero se considera una miasis frecuente en los ovinos de lana. Tampoco se encontraron reportes de caso de Gasterophilus sp. en equinos importados. No existen reportes de resistencia a insecticidas en miasis obligatorias en Colombia, aunque se encontró evidencia de resistencia a organofosforados por $C$. hominivorax en Brasil y Uruguay.

Es indispensable que el médico veterinario evite y rechace el uso de productos inapropiados para el tratamiento de las miasis (10). Algunos de los casos de miasis humana reportados en Colombia han sido en agricultores (11), pero es probable que exista un subregistro de casos en el país. El riesgo de miasis foruncular en humanos a partir de animales domésticos reservorio no ha sido evaluado en Colombia.

Finalmente, el médico veterinario debe estar atento a posibles acciones recíprocas entre las miasis y otras infecciones, como enfermedades causadas por priones (12), o interacciones antagónicas sobre nematodos gastrointestinales (13). Asimismo, es importante que se tomen en cuenta tratamientos alternativos contra las miasis (14), o incluso el uso de larvas en terapéutica veterinaria (15).

\section{Conclusiones}

En los países en desarrollo, y particularmente en Colombia, la información del sector público sobre la incidencia e impacto de las miasis en términos de mortalidad, morbilidad, costos de producción, resistencia a antiparasitarios, entre otros, se encuentra limitada. De hecho, las miasis obligatorias no son de notificación obligatoria ante los servicios veterinarios estatales en Colombia y puede existir un número elevado de casos humanos no registrados. Por lo anterior, las miasis humanas se pueden clasificar como enfermedades olvidadas (neglected diseases) que, no obstante, afectan con frecuencia a comunidades pobres con necesidades básicas insatisfechas. Bajo estas circunstancias, las condiciones sanitarias deficientes de las poblaciones de animales domésticos asociadas a dichas comunidades podrían favorecer el incremento de casos de miasis en las personas más vulnerables.

\section{Referencias}

1. Wall R, Shearer D. Veterinary ectoparasites: biology, pathology and control. Second editio. London: Blackwell Science Ltd.; 2001. p. 114-42.

2. Hope FW. On insects and their larvae occasionally found in the human body. Trans R Entomol Soc London. 1840;2:256-71.

3. Zumpt F. Myiasis in man and animals in the old world. London: Butheworths; 1965.

4. Caissie R, Beaulieu F, Giroux M, Berthod F, Landry PE. Cutaneous myiasis: diagnosis, treatment, and prevention. J Oral Maxillofac Surg. 2008;66:560-8.

5. Hall MJR, Smith KGV. Diptera causing myiasis in man. En: Medical insects and arachnids. Lane RP, Crosskey RW, editors. London: Chapman \& Hall; 1993. p. 429-69.

6. Lindsay R, Stancil J, Ray JM. Myiasis of facial wounds by Cochliomyia hominivorax sustained in a natural disaster in Haiti. Otolaryngol Head Neck Surg. 2010;143:595-6.

7. Cetinkaya M, Ozkan H, Köksal N, Coşkun SZ, Hacimustafaoğlu M, Girişgin O. Neonatal myiasis: a case report. Turk J Pediatr. 2008;50:581-4.

8. Moya-Borja GE. Erradicação ou manejo integrado das miíases neotropicais das Américas? Pesquisa Veterinária Brasileira. 2003;23:131-8.

9. Cramer-Ribeiro B, Sanavria A, Monteiro H, Oliveira M, Souza F. Inquiry of cases of myiasis by Cochliomyia hominivorax in dogs (Canis familiaris) of the Northern and Western zones of Rio de Janeiro city in 2000. Braz J Vet Res Animal Sci. 2003;40:13-20.

10. Forero-Becerra EG, Cortés-Vecino JA, VillamilJiménez LC. Factores de riesgo asociados a la miiasis por Cochliomyia hominivorax en fincas ganaderas de Puerto Boyacá (Colombia). Revista Científica, Universidad del Zulia, Venezuela. 2009;19:460-5.

11. Osorio J, Moncada L, Molano A, Valderrama S, Gualtero S, Franco-Paredes C. Role of ivermectin in the treatment of severe orbital myiasis due to Cochliomyia hominivorax. Clin Infect Dis. 2006;43:e57-9.

12. Lupi O. Myiasis as a risk factor for prion diseases in humans. J Eur Acad Dermatol Venereol. 2006;20:1037-45.

13. Angulo-Valadez CE, Scholl PJ, Cepeda-Palacios R, Jacquiet P, Dorchies P. Nasal bots... a fascinating world! Vet Parasitol. 2010;174:19-25.

14. Khater HF, Khater DF. The insecticidal activity of four medicinal plants against blowfly Lucilia sericata (Diptera: Calliphoridae). Int J Dermatol. 2009;48:492-7.

15. Jones G, Wall R. Maggot-therapy in veterinary medicine. Res Vet Sci. 2008;85:394-8. 


\title{
Gastroenteritis parasitarias en los animales domésticos
}

\author{
Jimmy Jolman Vargas \\ Grupo de Control Genético en Salud Animal, Instituto de Genética, \\ Universidad Nacional de Colombia, Bogotá, D.C., Colombia
}

Las gastroenteritis parasitarias que afectan a los animales domésticos, son enfermedades causadas por agentes infecciosos, en su mayoría macroparásitos, con parte de su ciclo de vida fuera de su huésped animal (1). Las formas larvarias de algunos artrópodos (Gasterophilus spp.), las larvas y los adultos de nematodos (Haemonchus contortus, Ancylostoma caninum, Strongylus vulgaris), de céstodos (Cysticercus tenuicollis, Moniezia expansa) y de trematodos (Fasciola hepatica, Paramphistimum cervi), así como las formas asexuales y sexuales de varios protozoarios (Giardia duodenalis, Isospora suis, Eimeria necatrix, Cryptosporidium parvum) continúan generando un impacto económico serio sobre las poblaciones animales, así como efectos sobre la salud pública en países tanto desarrollados como en desarrollo.

Los artrópodos, helmintos y protozoarios gastrointestinales causantes de enfermedades, son mucho más prevalentes en condiciones tropicales, donde las formas infecciosas pueden desarrollarse en contacto estrecho con los animales, especialmente cuando el manejo y la higiene son deficientes, situación bastante común en los sistemas productivos extensivos, informales o en aquéllos poco tecnificados (8).

El desarrollo, la supervivencia y la transmisión de las formas de vida libre de los diferentes parásitos causantes de gastroenteritis, están influenciados por factores ambientales, como la temperatura, la intensidad lumínica, la humedad, la pluviosidad, las cubiertas vegetales y las características del suelo; dichos factores combinados son responsables de las fluctuaciones en las prevalencias y cargas parasitarias.

En Colombia, el desarrollo de estadios infecciosos de parásitos es casi continuo durante todo el año con fluctuaciones poco marcadas, si se compara con zonas donde los cambios estacionales se encuentran bien establecidos; sin embargo, es probable que aquellas condiciones que permiten alcanzar rápidamente el estadio infeccioso limiten también la supervivencia de los mismos, debido a un mayor grado de actividad y a gasto de reservas energéticas. Conociendo esta biodinámica, ha sido posible establecer que en las condiciones tropicales los pequeños rumiantes con infecciones por nematodos Trichostrongylidos pueden pastorear de forma segura durante 4 a 5 días, tiempo necesario para alcanzar el estadio infeccioso, y pueden retornar 4 a 5 semanas después, tiempo necesario para agotar las reservas energéticas de las larvas infecciosas (6).

Con respecto a la transmisión, es claro que, a mayor disponibilidad de formas patógenas, mayor será la posibilidad de infección; por lo tanto, la densidad de las poblaciones, el tipo de instalación, los niveles de respuesta inmunológica por parte de los huéspedes, la edad, las condiciones nutricionales, el manejo y el estado productivo, son factores que juegan un papel determinante en la proliferación de los diferentes tipos de parásitos gastrointestinales. Frente a algunos de estos factores, que pueden limitar la biodisponibilidad de las formas infecciosas, los parásitos responden reprimiendo o inhibiendo su desarrollo en los huéspedes finales, como sucede en los casos de hipobiosis en nematodos o empleando huéspedes secundarios que protejan y favorezcan el desarrollo de las formas infecciosas (9).

En general, la mayoría de los parásitos gastrointestinales tienen ciclos monoxenos (un solo huésped) con formas infecciosas cuya resistencia a las condiciones medioambientales es variable; sin embargo, algunos parásitos gastrointestinales presentan ciclos heteroxenos (varios huéspedes), en los cuales las formas infecciosas, empleando artrópodos o moluscos, se protegen o se adaptan a condiciones ambientales adversas hasta alcanzar su desarrollo. El conocer adecuadamente la ecología de los parásitos gastrointestinales en sus formas de vida libre, en sus estadios infecciosos y en sus formas parásitas, es esencial en el diseño de los esquemas de control. Actualmente, los estudios en ecología molecular han permitido dilucidar procesos de transmisión e interacción huésped-parásito, con aplicaciones prácticas en términos de evolución, especiación y resistencia antihelmíntica (2)

Desde el punto de vista médico veterinario, el principal efecto clínico asociado a las parasitosis gastrointestinales es el síndrome diarreico agudo o crónico, el cual se manifiesta con un incremento en el contenido de agua en las heces comparado con la homeostasis, debido a los procesos inflamatorios intestinales causados por la presencia de parásitos. 
Las consecuencias de este síndrome pueden ser variables en términos de mortalidad y morbilidad $(4,7)$. Es posible detectar un incremento en las tasas de mortalidad en animales jóvenes o en especies muy sensibles; sin embargo, la mayoría de las parasitosis gastrointestinales animales inducen cuadros subclínicos crónicos, con manifestaciones como reducción del al nacer, destete prolongado, disminución de la tasa de ganancia de peso en los animales jóvenes, reducción en la fertilidad de los animales adultos, caída en el desempeño y baja producción de leche, lana y otros subproductos (3).

En la actualidad, existen múltiples herramientas para llevar a cabo el manejo preventivo de las infecciones parasitarias a nivel gastrointestinal, favoreciendo el bienestar y la salud tanto de los animales como del hombre, permitiendo vislumbrar logros más significativos en cuanto a su prevención y control. Un programa de manejo adecuado de las parasitosis gastrointestinales, implica limitar toda repercusión económica, a partir de la obtención de un beneficio óptimo. El control de las parasitosis gastrointestinales mediante programas de erradicación, no es una medida práctica; normalmente, el control debe estar enmarcado en el uso combinado de tratamientos antihelmínticos y prácticas de manejo ambiental.

Los medicamentos antihelmínticos son eficientes en el control de cuadros clínicos agudos y reducen la mortalidad debida a las infecciones parasitarias; sin embargo, no previenen la exposición de los animales a medios ambientes muy contaminados con formas infecciosas y, en los últimos años, se ha presentado un incremento importante en el desarrollo de la resistencia a estos medicamentos por parte de las poblaciones de parásitos (5).

Debido a que las estrategias de control deben ser complementarias, la selección de animales domésticos resistentes a las infecciones parasitarias es una alternativa viable que contribuye a mejorar el estatus sanitario y el desempeño de las poblaciones animales. Es necesario diferenciar claramente los procesos de tolerancia, resistencia y resilience en las poblaciones animales, para lograr cambios epidemiológicos significativos en las tasas de infección. La selección genética de animales resistentes es una alternativa viable; sin embargo, se requiere comprender mejor las diferencias genéticas relacionadas con la respuesta inmunitaria generada frente a los agentes parasitarios (5).
Todas las aproximaciones relacionadas con el control y la prevención de las gastroenteritis parasitarias requieren de herramientas diagnósticas y de vigilancia adecuadas, que permitan cuantificar el efecto de cada medida; de esta forma, el uso de las herramientas de diagnóstico coproparasitario y el desarrollo de nuevos marcadores moleculares, facilitan cada día más esta tarea.

Asimismo, la caracterización de modelos para el estudio de las gastroenteritis parasitarias en animales, es un campo muy amplio que día a día adquiere mayor importancia y relevancia, si se tiene en cuenta la necesidad de comprender mejor los complejos procesos inmunológicos y ecológicos asociados a la resistencia, así como la dinámica de las poblaciones de parásitos, el desarrollo de nuevos mecanismos de infección y el advenimiento de la resistencia a las moléculas empleadas para su control.

\section{Referencias}

1. Bishop S. International course in study of resistance mechanisms in animal infectious diseases. Liège: EADGENE; 2009.

2. Criscone CD, Poulin R, Blouin MS. Molecular ecology of parasites: elucidating ecological and microevolutionary processes. MolEcol.2005;14:224757.

3. Scott P. Health and production management in sheep flocks. In: Herd health. Food animal production medicine. Third Edition. 2001. Philadelphia: W. B. Saunders Company. p. 766-844.

4. Stampfli $\mathrm{H}$, Oliver O. Chronic diarrhea and weight loss in tree horses. Vet Clin Equine. 2006;22:27-35.

5. Vanegas D, White IM, Stear MJ, Bishop S. Estimation of heritabilities and correlations between repeated faecal egg count measurements in lambs facing natural nematode parasite challenge, using a random regression model. J Agricultl Sci. 2007;145:501-8

6. Vargas JJ. Pautas a seguir en un programa de control parasitario en una explotación ovina y caprina. Memorias, II Congreso Internacional Ovino y Caprino, Bogotá, D.C., 2005.

7. Vargas JJ. Síndrome diarreico asociado a parásitos en equinos. Memorias, III Seminario Internacional de Enfermedades Parasitarias en Bovinos y Equinos, Bogotá, D.C, 2007.

8. Vargas JJ, Vega JC. Manual de parasitología veterinaria. Guías de práctica. Bogotá: Cortés-Duarte Editores; 2007.

9. Vargas JJ, Vega JC, Forero E, Cortes JA. Parasitología veterinaria. Fundamentos y prácticas. Bogotá: Papel y Plástico Ltda.; 2010. 


\title{
Babesiosis bovina, la verdadera "fiebre de garrapatas", gran limitante para una óptima productividad ganadera en el trópico
}

\author{
Otoniel Vizcaíno-Gerdts \\ Laboratorios LIMOR de Colombia, Bogotá, D.C., Colombia
}

\section{Introducción}

La babesiosis bovina, enfermedad hemolítica, febril, anemizante y hemoglobinúrica, produce pérdidas económicas considerables por muertes, por disminución de la productividad y por gastos en insumos para su control. Se considera como la enfermedad con el impacto económico más importante de los bovinos en áreas tropicales del mundo $(1,2)$. Es ocasionada especialmente por la Babesia bovis y Babesia bigemina, protozoos que tienen igual distribución a la de su principal vector, Rhipicephalus (Boophilus) microplus, en regiones situadas entre los $32^{\circ} \mathrm{S}$ y $40^{\circ} \mathrm{N}$ de la línea del Ecuador. Actualmente, por el calentamiento global, esta distribución es más amplia. La enfermedad produce mayores pérdidas en países en desarrollo cuando no se ha determinado muy bien la epidemiología de los organismos ni de sus vectores, ni se adoptan alternativas eficaces de control ya existentes (culturales, químicas, inmunológicas).

\section{Antecedentes históricos}

En 1906 murieron, aproximadamente, 3’000.000 de bovinos $(54,5 \%$ de la población bovina de Queensland) (3), con síntomas clásicos de babesiosis, los cuales había observado Babes en Rumania con Babesia bovis en 1888 y Smith y Kilbourne en 1893 con Babesia bigemina en los Estados Unidos (4). En Colombia se adelantaron importantes trabajos sobre babesiosis y anaplasmosis en el marco de los convenios internacionales del Instituto Colombiano Agropecuario con universidades estadounidenses (Texas $A \& M$ e Illinois) y con el Convenio Colombo-Alemán en las décadas de 1960 a 1980 (5-10). En el 2000, el Instituto Colombiano Agropecuario aprobó la vacuna Anabasan®-LIMOR, para prevenir a los bovinos de la anaplasmosis y las babesiosis (11).

\section{Especies de babesias bovinas y sus vectores}

Se han descrito básicamente cinco especies de babesias bovinas las cuales se diferencian por tamaño, por capacidad patógena, por identidad serológica y por el vector que las transmite. Últimamente se utilizan patrones enzimáticos, antigénicos y por ADN de la especie, entre ellas Babesia bovis, Babesia bigemina, Babesia divergens, Babesia major y Babesia jalimovi.

\section{Epidemiología de las babesiosis bovinas}

Tienen una amplia distribución en las áreas intertropicales del mundo donde es prevalente su principal vector, la garrapata Rhipicephalus (Boophilus) microplus donde la mayoría de la población bovina del mundo estimada en 1.371 billones, se encuentra potencialmente a riesgo de las babesiosis. Los factores que limitan la distribución de los artrópodos vectores lo constituyen la humedad y la temperatura, factores que tienen una estrecha relación inversamente proporcional con la altitud. Otros factores importantes que inducen brotes de babesiosis, lo constituyen el incremento de razas Bos taurus en áreas endémicas de garrapatas sin previa inmunoprevención, la aparición del fenómeno de resistencia de las garrapatas o los acaricidas y, actualmente, la influencia del cambio climático el cual favorece el desplazamiento de las garrapatas a áreas de mayor altitud donde existe una población bovina más susceptible a los hemoparasitismos. Considerando la apreciación de Mahoney de 1962, las explotaciones bovinas se encuentran en un estado de "estabilidad enzoótica" con rara presentación de casos clínicos, cuando la tasa de inoculación de vermículos de babesia es igual o mayor de 0,005. En Colombia, en tres importantes regiones ganaderas, Corrier realizó estudios epidemiológicos de prevalencia serológica de hemoparasitismos en 1975: la región Caribe, la Orinoquia y la región del Pacífico.

- Región Caribe (28 ${ }^{\circ} \mathrm{C}$ y $13-15$ msnm). La región la integran siete departamentos, los cuales suman una población bovina aproximada de 7’465.738 bovinos. La prevalencia serológica para Babesia bigemina en la década de los 70 en Córdoba y Sucre fluctuó en 68 \% y la infección en pastoreo se presentó a las dos semanas y para Babesia bovis, la transmisión natural en un ternero esplenectomizado ocurrió a los 7 días de pastoreo.

- La región de la Orinoquia (departamento del Meta) $\left(26^{\circ} \mathrm{C}\right.$ y $\left.450 \mathrm{msnm}\right)$ está integrada por seis departamentos que suman, aproximadamente, 3’354.496 bovinos. En el área del Meta en la década 70 , se realizaron estudios epidemiológicos de hemoparasitismos. Para Babesia bigemina los reactores positivos fue de $42 \%$ y en la década 
de los 80 la prevalencia fue de $84 \%$ y 72,8 $\%$ respectivamente para Babesia bigemina y para Babesia bovis en un estudio realizado por Mateus en 1987. La garrapata más importante por su frecuencia y distribución es también $R$. (B.) microplus.

- La región del Pacífico (Valle del Cauca) $\left(24^{\circ} \mathrm{C}\right.$ y $1.000 \mathrm{msnm}$ ) la integran cinco departamentos que suman, aproximadamente, 3'789.349 bovinos, más susceptibles que la de las regiones Caribe y la Orinoquia por su mayor proporción de sangre Bos taurus. Ocasionalmente, se presenta mortalidad que supera el $50 \%$ cuando movilizan el ganado en épocas de escases de pastos a regiones de menor altitud con presencia de garrapatas. Acostumbran, por temor a las garrapatas, a mantener a los terneros en confinamiento hasta los 6 a 10 meses de edad, los cuales enferman en las primeras semanas de pastoreo. La prevalencia para Babesia bovis y Babesia bigemina en la década de los 70 fluctuó entre $46,3 \%$ y $81,1 \%$ respectivamente, según los estudios realizados por González et al. en 1978. La práctica de bañar los animales en periodos menores a su periodo biológico de multiplicación, incrementa la susceptibilidad a los hemoparasitismos. Igualmente, $R$. (B.) microplus es la garrapata de mayor prevalencia.

\section{Impacto económico de las babesiosis}

Las pérdidas deben estimarse considerando una suma de gastos: terapia química de microorganismos (profiláctica y terapéutica), gastos en tratamientos sintomáticos, en baños garrapaticidas, pérdidas ocasionales por abortos, por disminución de la productividad, gastos en alternativas inmunoprofilácticas contra el vector y contra los organismos que transmiten y gastos por servicios veterinarios y por diagnóstico de laboratorio.

Anotemos algunos ejemplos: La FAO en 1988 estimó pérdidas por US\$7.000 millones por año en 1.200 millones de bovinos en áreas tropicales del mundo ocasionadas por hemoparasitismos y sus vectores, en los Estados Unidos, US\$ 500 millones al año; en el Reino Unido el costo por Babesia divergens asciende a US\$800.000 al año; en Queensland, Australia, US\$ 644.000 por año, y en América Latina, US\$ 875 millones por año, el costo de los hemoparasitismos y sus vectores.

\section{Control químico de las babesiosis}

Para una terapia específica por babesiosis, se debe recurrir básicamente a dos tipos de medicamentos: aceturato de diminazeno y dipropionato de imidocarb (12).

\section{Protección inmunológica de las babesiosis}

Actualmente, existen algunos pocos países con soluciones inmunológicas, entre ellos Colombia (13) para el control de babesias y para la rickettsia anaplasma, basadas en el método australiano.

\section{Referencias}

1. McCosker. The global importance of babesiosis. In: Ristic M, Kreier J, editors. Babesiosis. New York: Academic Press; 1981. p. 1-24.

2. Babesiosis bovina. Fiebre por garrapatas, Fiebre de Tejas, Fiebre hematúrica. The Center for Food Security \& Public Health, Institute for International Cooperation Animal Biologics, OIE. 2010.

3. Tick Fever Research Centre. 25 years of service. 1966-1991.

4. Smith, Kilbourne. Investigations into the nature; causation and prevention of Texas or southern fever. Washington, D.C.; 1893.

5. Vizcaíno GO, Thompson K, Mateus G. Aislamiento de Babesia argentina (Sin. B. bovis) utilizando larvas de Boophilus microplus. Memorias, III Congreso Nacional de Medicina Veterinaria y Zootecnia, Cúcuta, 1971.

6. Bishop JP, Adams LG, Thompson KC et Corrier DE. The isolation, separation and preservation of Babesia bigemina. Trop An Hlth Prod. 1973;5:141-5.

7. Todorovic RA, Vizcaíno GO,Adams LG. Determinación de anticuerpos de babesia para la técnica de fijación del complemento. Revista ICA. 1971;6:221-33.

8. Todorovic RA, Vizcaíno GO, González EF, Adams LG. Chemoprophylaxis (Imidocarb) against Babesia bigemina and Babesia argentina infections. Amer J Vet Res. 1973;34:1153-61.

9. Vizcaíno GO, Todorovic RA. Caracterización de los antígenos de Babesia argentina (sin. B. bovis) y Babesia bigemina por los métodos de fijación del complemento, inmunodifusión, inmunoelectroforesis e Inmunidad cruzada. Revista ICA. 1975;10:77-85.

10. González EF, Todorovic R, López G, y García O. Atenuación de una cepa de Babesia argentina (Babesia bovis) de origen colombiano. Revista ICA. 1979;14:33-9.

11. Vizcaíno GO, Benavides OE. Vacuna Anabasan®, alternativa colombiana para prevenir el ganado bovino de la anaplasmosis y las babesiosis. Revista ACOVEZ. 31: 7.

12. Vial HJ, Gorenflot. A Chemotherapy against babesiosis. Vet Parasitol. 2006;138:147-60.

13. Cantó AGJ, Álvarez MJA, Rojas REE, Ramos AJA, Mosqueda GJJ, Vega MCA, Figueroa MJV. Protección contra babesiosis bovina con una vacuna mixta de Babesia bovis y Babesia bigemina derivada de cultivo in vitro bajo una confrontación de campo Inmunización en un área libre de la enfermedad. Vet Mex. 2003;34:323-32. 


\title{
Parasitología veterinaria y cambio climático
}

\author{
Jesús Antonio Betancourt \\ Bogotá, D.C., Colombia
}

\section{Introducción}

El cambio climático es un fenómeno ya reconocido a nivel mundial; algunos de sus componentes que lo hacen evidente y que están aumentando en magnitud y significancia, según Pollary, et al. (2010), son:

- el calentamiento,

- los patrones alterados de precipitación y

- la incidencia aumentada de eventos climáticos extremos.

Colombia no es ajeno al fenómeno y a sus implicaciones en variabilidad climática (Pabón y Torres, 2007), en salud humana (Poveda, et al., 2000) y animal (Betancourt, 2010). CORPOICA está ya ejecutando proyectos, con la cofinanciación del Ministerio de Agricultura y Desarrollo Rural, de mitigación y adaptación a los efectos del cambio climático. (Ayarza, et al., 2008).

\section{Las enfermedades parasitarias y el cambio climático}

Aunque el cambio climático puede afectar la distribución y el comportamiento de enfermedades de diversa etiología, se considera que las enfermedades transmitidas por artrópodos $-\mathrm{y}$ los vectores mismos- son altamente sensibles a cambios en las condiciones climáticas (Kovats, et al., 2001). El cambio climático puede alterar la relación de un animal con parásitos y vectores.

No obstante, la aparición y diseminación de enfermedades parasitarias, y de otras transmitidas por vectores, no pueden atribuirse exclusivamente al cambio climático. Harrus y Banet (2005) han listado varios factores bióticos y abióticos que intervienen en la distribución y en el comportamiento de plagas y enfermedades.

Adicionalmente, Kovats, et al. (2001) presentan como requerimientos mínimos para aceptar una relación causal entre el cambio climático y los cambios en salud, los siguientes:

- evidencia de sensibilidad biológica al clima,

- evidencia meteorológica de cambio climático y

- evidencia de cambio entomológico.

La supervivencia y la capacidad vectorial

Se asume que el calentamiento global favorecerá a los parásitos y llevaráal aumento de enfermedades debido a que las tasas de los procesos fisiológicos de la mayoría de los invertebrados son dependientes de la temperatura ambiente (poiquilotermos) (Wall y Morgan, 2009). Estos autores conceptúan que el número resultante aumentado de generaciones por año y los períodos prolongados favorables para transmisión incrementarán la abundancia temporal de parasitosis endémicas. Al respecto, Sánchez (2009) anota que en España -y, posiblemente, en otros países de Europa- los inviernos cada vez más cálidos favorecen las actividades de los parásitos y permiten que sean activos durante todo el año y no solamente durante los meses de calor, aumentándose el riesgo de transmisión. Statz (2007) manifiesta que los parásitos animales están avanzando más y más hacia áreas templadas de Europa y Norteamérica desde regiones cálidas, como el Mediterráneo y Centroamérica.

\section{Efectos económicos}

Pocos han tratado el tema de los posibles efectos económicos de la colonización de regiones. White, et al. (2003) emplearon un modelo de poblaciones de garrapatas combinado con un sistema de información geográfica (SIG) en diferentes condiciones climáticas y lo relacionaron con estadísticas de la industria cárnica de Australia. El estudio evidenció cambios en abundancia y en expansión de poblaciones de garrapatas y reducción en productividad. Sin medidas de adaptación, las pérdidas en ganancia de peso en el año 2030, serían de 7.780 toneladas al año y en el año 2100 serían de 21.637 toneladas al año, frente a las pérdidas actuales que son de 6.594 toneladas por año.

\section{Artrópodos y las enfermedades que transmiten}

Difícilmente se pueden tratar por separado la expansión de diferentes artrópodos parásitos de los animales y del hombre, y la de las enfermedades de las cuales son vectores o reservorios. Uno de los artrópodos que más ha sido objeto de estudio en cuanto a su desplazamiento a nuevas áreas es la garrapata. Estrada-Peña (2001), empleando información meteorológica satelital y el índice de vegetación normalizado, estudió el tema con relación a Rhipicephalus (Boophilus) microplus en Centroamérica, y halló una alta favorabilidad para esta especie en zonas del sureste de los Estados Unidos, la mayor parte de México y otras áreas de Centroamérica. El trabajo indicó el establecimiento de nuevos focos en zonas que han 
sido consideradas "demasiado frías" para sostener poblaciones de garrapatas.

Hallazgos similares han sido reportados por Cortés, et al. (2010) en el altiplano cundiboyacense de Colombia, donde encontraron físicamente $R$. (B.) microplus parasitando bovinos en altitudes hasta de $2.903 \mathrm{msnm}$, cuando se consideraba -40 años atrás (Evans, 1978)- que esta garrapata difícilmente podría establecerse por encima de los $2.400 \mathrm{msnm}$.

Los estudios con modelos basados en información meteorológica provista por Wordclim y con ayuda de programas, como MaxenT y GARP, realizados por Pulido, et al. (2011), presentan la expansión actual y futura (año 2020) en altitud y latitud de $R$. (B.) microplus y de un insecto plaga de los pastos (Collaria scenica), en esta región alta del país. En concordancia con esto, los estudios realizados por Corpoica, tanto parasitológicos como serológicos, han indicado el incremento en prevalencia de hemoparásitos de bovinos.

Europa, Estados Unidos y Canadá han documentado expansión de garrapatas, principalmente hacia el norte, junto con sus patógenos asociados. Ixodes ricinus se ha diseminado al norte de Suecia, de los 61 a los $66^{\circ} \mathrm{N}$. En la República Checa, los estudios realizados en 1957 y 1979 detectaron $I$. ricinus, hasta 700 msnm; en los años 2000 y 2002, la especie fue recolectada en perros a $1.100 \mathrm{msnm}$, en áreas donde antes estaba ausente. Dermacentor reticulatus ha extendido su rango en Alemania; Hyalomma marginatum se está extendiendo al norte de España y Rhipicephalus sanguineus habitualmente limitada al sur de Europa, puede migrar hacia el norte por temperaturas más cálidas (CABI blogs, 2009).

Greifenhagen y Nolan (2003) reportaron la migración hacia el norte del Canadá, de Ixodes scapularis, vector de la enfermedad de Lyme. También reportaron la migración de Dermacentor variabilis y Dermacentor andersoni, vector de Rickettsia rickettsi. Los mismos autores, sugirieron la posibilidad de una migración hasta Ontario de $R$. sanguineus, vector de erlichiosis canina.

En el África subsahariana, Olwoch, et al. (2008) predijeron el impacto del cambio climático en el rango de distribución de Theileriosis, usando modelación. Los resultados, basados en la distribución proyectada de Rhipicephalus appendiculatus, muestran aumento de favorabilidad para esta garrapata y para teileriosis Sudáfrica, Botswana, Malawi, Zambia y la República Democrática del Congo.
Los insectos, al igual que las garrapatas, también han presentado cambios en su distribución, Ilevando con ellos sus patógenos. Tal es el caso de Culex pipiens, vector de la enfermedad del Nilo oriental en los Estados Unidos y de Culicoides imicola, vector de lengua azul en Europa. Igualmente, Phlebotomus spp. parece haber extendido su distribución en el norte de España causando leishmaniasis canina en una región a $1.000 \mathrm{msnm}$, donde la enfermedad era desconocida 20 años atrás.

El cambio climático parece estar afectando la distribución de insectos asociados directamente con patologías, como son las miasis cutáneas. Wall yEllse (2011) emplearon un modelo para proyecciones de miasis cutáneas en ovejas en el Reino Unido y encontraron una estación prolongada de miasis y más incidencia acumulativa del problema. Estos autores estimaron que un aumento de $3{ }^{\circ} \mathrm{C}$ en temperatura media, puede duplicar la incidencia acumulativa de las miasis en corderos y cuadruplicar el problema en ovejas. Trabajos actualmente en ejecución en Corpoica, detectaron bovinos infectados con larvas de Dermatobia hominis en altitudes hasta de 2.350 msnm; este hallazgo se considera inusual, dado que el parásito mencionado ha sido reportado principalmente en zonas de ladera por debajo de los $2.000 \mathrm{msnm}$.

Los reportes de la OMS (2000) y de FAO-IAEA (2010) incluyen las siguientes enfermedades parasitarias animales, transmitidas por artrópodos, entre las que están expandiendo su distribución, posiblemente en asocio con el cambio climático en diferentes regiones del mundo:

\begin{tabular}{|c|c|}
\hline - Tripanosomosis bovina & $\begin{array}{l}\text { África, } \\
\text { Suramérica* }\end{array}$ \\
\hline - Enfermedad de Lyme & $\begin{array}{l}\text { Europa, } \\
\text { Norteamérica }\end{array}$ \\
\hline - Leishmaniasis & $\begin{array}{l}\text { Europa, } \\
\text { Suramérica }\end{array}$ \\
\hline $\begin{array}{l}\text { - Erlichiosis monocítica } \\
\text { canina }\end{array}$ & $\begin{array}{l}\text { Norteamérica, } \\
\text { Europa (E. canis) }\end{array}$ \\
\hline $\begin{array}{l}\text { - Erlichiosis trombocítica } \\
\text { canina }\end{array}$ & $\begin{array}{l}\text { Europa, } \\
\text { Suramérica }(A . \\
\text { platys) }\end{array}$ \\
\hline $\begin{array}{l}\text { - Enfermedad de Chagas } \\
\text { - Anaplasmosis }\end{array}$ & $\begin{array}{l}\text { Suramérica } \\
\text { Norteamérica y } \\
\text { Suramérica }\end{array}$ \\
\hline - Babesiosis canina & $\begin{array}{l}\text { Suramérica, } \\
\text { Europa }\end{array}$ \\
\hline $\begin{array}{l}\text { - Babesiosis bovina } \\
\text { - Fiebre de garrapatas, ovina }\end{array}$ & $\begin{array}{l}\text { Suramérica* } \\
\text { Inglaterra, } \\
\text { Escocia. }\end{array}$ \\
\hline
\end{tabular}


A lo anterior se suman las enfermedades transmitidas por pulgas (Ctenocephalides felis, $C$. canisPulex irritans y Xenopsylla cheopis) como:

- Arañazo de gato, causada por Bartonella

- Micoplasmosis caiusada por Haemoplasma haemominutum, Mycoplasma haemofelis y Mycoplasma haemocanis

- Rickettsiosis causada por Rickettsia felis (Beugnet y Marie, 2009).

\section{Cryptosporidium spp. y Giardia spp.}

Según Pollary, et al., (2010), el cambio climático tieneel potencialdealterarlastasas desupervivencia para ooquistes y quistes; ya que ambos estadios son hallados en aguas de superficie. Los cambios en la hidrología regional y local pueden alterar la distribución de estos parásitos y, a su vez, el riesgo de exposición humana y animal.

\section{Helmintos}

Los helmintos parecen no escapar a los efectos del cambio climático. El más frecuentemente mencionado en este sentido es Fasciola hepática. Mientras que a $15^{\circ} \mathrm{C}$, los huevos eclosionan en 80 días, a $25^{\circ} \mathrm{C}$, lo hacen en sólo 10 días. Además, se requiere un ambiente húmedo para que los huevos eclosionen, el miracidio contacte al caracol y las cercarías migren al pasto y se enquisten en él. (Skuce, 2008). Una publicación de FAO-IAEA (2010) anota que ahora se pueden observados los caracoles intermediarios de $F$. hepática, en varias áreas por encima de los 4.200 msnm en tierras altas de Perú y Bolivia. Rundle (2011) combinó técnicas de predicción de enfermedades con proyecciones de cambio climático y sugirió que para el año 2020, en Escocia se podrían presentar serias epidemias de fascioliasis, con episodios clínicos graves en el país de Gales para el año 2050. La mayor parte del oeste de Inglaterra también se vería afectada.

En cuanto a los nematodos causantes de las parasitosis gastrointestinales, un análisis de los datos del período 1993-2008, muestra un aumento del diagnóstico de tal parasitismo en ovinos en Escocia. El género Haemonchus, considerado antes raro en Escocia, parece que actualmente es endémico. Teladorsagia circumcincta, antes considerada también rara en el Reino Unido, es ahora el parásito de ovejas más importante en ese territorio. Se cree que el parásito cambió en estacionalidad y que ahora se diagnostican rutinariamente grandes infestaciones en corderos en primavera. Nematodirus battus, parásito intestinal de ovinos que se presentaba en corderos jóvenes tarde en primavera y temprano en verano en el Reino Unido, parece también haber cambiado de estacionalidad, ya que ahora es visto en corderos más viejos en otoño e invierno.

La revisión de los datos meteorológicos de 30 años, junto con las parasitosis gastrointestinales en ovejas y bovinos, dieron al cambio climático como la explicación más probable para los patrones de parasitismo observados.

En Colombia parecen existir indicaciones en el sentido de que parásitos del género Cooperia en bovinos, reportado anteriormente como más frecuente en climas medios y cálidos (Parra y Uribe, 1990; Thullner, et al., 1991), hoy parece predominar en zonas del altiplano, por encima de 2.550 msnm, donde otrora Ostertagia predominaba (Márquez, et al., 2000). Según Genchi, etal., (2011), el cambio climático y un aumento de reservorios (principalmente, perros infectados), han causado variación en el rango geográfico de Dirofilaria repens, desde áreas tradicionalmente endémicas del sur de Europa, hacia el norte del continente.

\section{Comentario final}

Aun teniendo presente que los cambios en la distribución de especies de parásitos, no son exclusivamente debidos al cambio climático, los reportes de la literatura sobre su expansión, más allá de sus latitudes habituales (en regiones templadas) y más arriba de sus altitudes habituales (en regiones tropicales e intertropicales), son cada día más numerosos e incluyen cada vez más especies de parásitos.

Las migraciones de artrópodos vectores de enfermedades hacia regiones donde antes no eran frecuentes, pueden introducir patógenos contra los cuales las especies domésticas o silvestres nativas de las regiones invadidas, no tienen una respuesta inmune previamente montada, resultando en episodios de enfermedad clínica.

Si bien, herramientas como los sistemas de información geográfica, la modelación y la geoestadística pueden ayudar a proyectar la distribución de plagas, se requiere la actualización del conocimiento epidemiológico y entomológico de las nuevas regiones para aumentar la precisión de los modelos.

\section{Bibliografía}

1. Ayarza M, Lascano C, BetancourtA, PeñaA, Cárdenas E. Revista Innovación y Cambio Tecnológico. 2008;7:54-9.

2. Betancourt A. Vectores en la salud animal y sus correlaciones con el cambio climático global. Seminario Internacional sobre Cambio Climático Global y Ganadería. Bogotá, 20-21 de mayo, 2010.

3. Beugnet F, Marie JL. Vet Parasitol. 2009;163:298305. 
4. CABI Blogs. Ticks in a changing climate, 2009.

5. Cortés JA, Betancourt JA, Argüelles J, Pulido A. Revista Corpoica Ciencia y Tecnología Agropecuaria. 2010;11:73-84.

6. Estrada-Peña A. J Parasitol. 2001;87:978-87.

7. Evans DE. Thesis. London: University of East London; 1978.

8. FAO-IAEA. Joint FAO-IAEA Programme. Nuclear Techniques in Food and Agriculture, 2010.

9. Genchi C, Kramer L, Rivasi F. Vector borne and zoonotic diseases, 2011, en prensa.

10. Greifenhagen S, Noland TL. Font Research Information Paper № 154. Ontario Font Research Institute; 2003.

11. Harrus S, Baneth G. Intl J Parasitol. 2005;35:130918.

12. Kovats RS, Campbell-Lendrum DH, McMichael AJ, Woolward A, Cox J. Trans Roy Soc London B. 2001;356:1057-68.

13. Lucientes J. Revista Albeitar. 2007;104:4-11.

14. Márquez DA, Jaramillo F, Romero A. Revista de Medicina Veterinaria y Zootecnia. 2000;47:49-56.

15. Olwoch JM, Reyers B, Engelbrecht F, Erasmus BFN. J Arid Environ. 2008;72:108-20.

16. Pabón JD, Torres G. Cuadernos de Geografía. 2007;16:81-94.
17. Parra D, Uribe LF. Epidemiología de nematodos del bovino en el piedemonte de los Llanos Orientales de Colombia. Revista ACOVEZ. 1990;14:16-25.

18. Pollary L, Hoberg E, Kutz S. Acta Vet Scand. 2010;52(Supp.1):S1.

19. Poveda G, Graham NE, Epstein P, Rojas W, Quiñones ML, Vélez ID, Martens WJ. Cambridge: Cambridge University Press; 2000. p.183-204.

20. Pulido LA, Betancourt JA, Osorio $P$, Barreto $N$, Argüelles J. XXXVIII Congreso, Sociedad Colombiana de Entomología, Manizales, 27-29 de julio. 2011.

21. Rundle K. Scottish Agricultural College; 2011.

22. Sánchez S. CVBD Forum en Sevilla. Forum Bayer. Comunicado de Prensa 24.03.2009.

23. Skuce PJ.. Scottish Animal Health and Welfare Conference. Dunkeld, Nov. 28, 2009.

24. Statz K. Bayer Health Care. 2007.

25. Thullner F, Roqueme L, Parra GD, Otte J. GTZ-ICA. Informe Técnico № 10, 1991.

26. Wall R, Morgan E. Vet Parasitol. 2009.

27. Wall R, Ellse LS. Global Change Biology. $2011 ; 17: 1770-7$

28. White N, Sutherst RW, Hall N, Whish-Wilson P. Climate Change. 2003;61:157-90.

29. WHO. Bull World Health Org. 2000;78. 


\title{
Simposio \\ SaLUd Geoespacial
}

\section{Leveraging spatial data on vector distribution for disease risk assessments}

\author{
Desmond H. Foley, Richard C. Wilkerson, Pollie L.M. Rueda
}

Division of Entomology, Walter Reed Army Institute of Research, Silver Spring, MD, USA

VectorMap (www.vectormap.org) is a public
access online spatial database of mosquito,
sand fly, and tick species collection records and
distribution models that is aimed at medical
entomologists, vector disease control workers,
preventative medicine practitioners, and health planners. VectorMap is an outgrowth of MosquitoMap (Foley, et al., 2010) that currently hosts around 250,000 mosquito records. Data is checked for geographic and taxonomic errors, and is comprised of vouchered specimen information, and both unpublished and unpublished observation data. VectorMap uses Microsoft Silverlight ${ }^{\mathrm{TM}}$ and ESRI's ArcGIS Server $10^{\mathrm{TM}}$ software platform to present disease vector data and relevant remote sensing layers in an online GIS format. Users can view the locations of past vector collections and the results of models that predict the geographic extent of individual species. Collection records are searchable and downloadable, and Excel collection forms with drop down lists, and Excel charts to country, are available for data contributors to map and quality control their data (Foley, 2011).

An application under development -the Mal-area Calculator (MAC)-allows the quantification, for any area of interest, of the overlap of vectors, hosts, and disease, to enable a first order approximation of vector hazard, and to fine-tune vector-borne disease maps. The Mal-area approach emphasizes the independent but cumulative contribution to disease risk of the vector species predicted present. VectorMap and the MAC makes accessible, and adds value to, the results of past vector collecting efforts. These online resources are offered to the

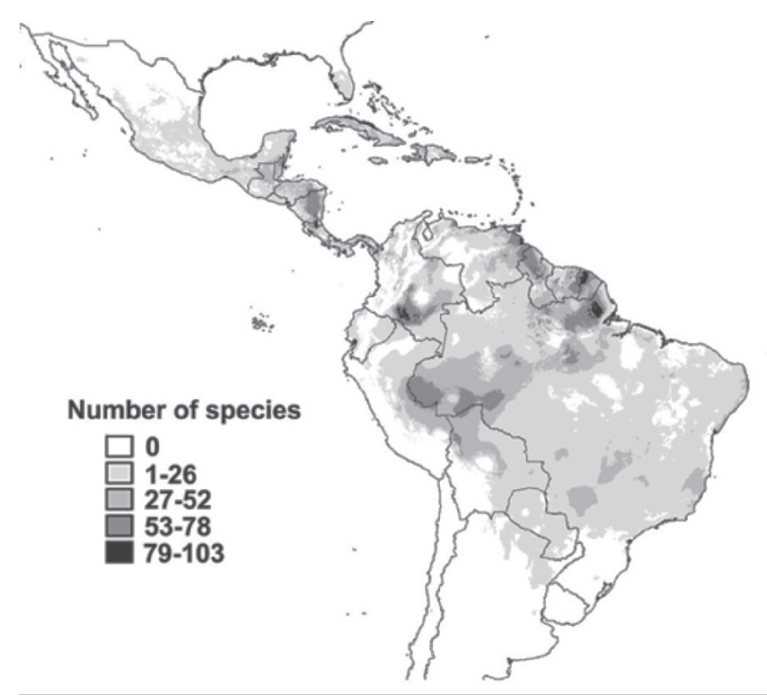

Figure 1. Map of mosquito species richness created by summing models (Bioclim) of species potential distribution that were created from collection records available in VectorMap.

international vector control community as a focal point to increase collaboration and to explore the nexus between geography and vector borne disease transmission.

\section{References}

1. Foley DH, Wilkerson RC, Birney I, Harrison S, Christensen J, Rueda LM. MosquitoMap and the Mal-area calculator: new web tools to relate mosquito species distribution with vector borne disease. Int $\mathrm{J}$ Health Geogr. 2010;9:11

2. Foley $\mathrm{DH}$. A spreadsheet mapping approach for error checking and sharing collection point data. Biodiversity Informatics. 2011;7:137-42. 